\title{
Avaliação da Aplicabilidade de um Modelo de Qualidade para Especificações de Projetos Orientados a Objetos
}

\author{
Denia K. Rezende ${ }^{a}$, Walcélio L. Melo ${ }^{b}$ \\ ${ }^{a}$ Universidade Católica de Brasília, Faculdades Alfa, deniakuhn@ cultura.com.br \\ ${ }^{\mathrm{b}}$ Universidade Católica de Brasília, walcelio.melo@computer.org
}

\begin{abstract}
Resumo
Os modelos de qualidade surgiram como uma forma de identificar fatores de qualidade que são importantes e que precisam ser avaliados durante o processo de desenvolvimento do software, fornecendo subsídios para o gerente de projeto tomar decisões importantes, minimizando riscos e buscando a garantia da qualidade desse produto. O objetivo deste documento é avaliar a aplicabilidade de um modelo de qualidade voltado para as especificações orientadas a objetos. Para avaliar tal modelo, realizamos quatro estudos de casos de sistemas comerciais e governamentais, desenvolvidos segundo uma metodologia orientada a objetos. O modelo de referência utilizado foi o Manual para Controle da Qualidade de Especificações Orientadas a Objetos, proposto em Beaufond [1]. Esse modelo utiliza o método de avaliação proposto por Rocha [2]. São mostradas aqui as razões que nos levaram a adotar o Manual. Também apresentamos uma lista de questões que foram levantadas referentes à aplicação desse Manual durante o processo de avaliação, obtendo como resultado seus aspectos positivos e negativos.
\end{abstract}

Palavras-chave: Qualidade de Software, Métricas de Software, Métricas com Indicadores de Limite, Especificações orientadas a objetos, Modelos de qualidade.

\begin{abstract}
Software quality models emerged as a way of identifying and evaluating important quality factors that need to be evaluated during the software developmental process. These models can supply project managers with design-making information allowing them to take important decisions to minimize risks. The goal of this paper is to evaluate the applicability of a quality model for object-oriented specifications called "Manual para Controle da Qualidade de Especificações Orientadas a Objetos" (manual for quality control of objectoriented specifications) proposed by Beaufond [1]. This model relies on the evaluation method proposed by Rocha [2]. For evaluating such model case studies were carried out. The model of reference utilized was "Manual para controle da Qualidade de Especificações Orientada a Objeto" (manual for quality control of object-oriented specifications) proposed by Beaufond [1]. The reasons that led us to adapt the formerly mentioned manual are shown here. A list of questions that were raised during the process of evaluation is presented.
\end{abstract}

Keywords: Software quality, Software metrics, Thresholds, Object-oriented specifications, Quality model.

\section{Introdução}

Para avaliar um conjunto de características ou atributos que envolvem a qualidade do software em vários níveis, é necessário escolher um modelo que organize esses atributos e permita 
avaliar e entender como as diferentes visões resultantes contribuem para a qualidade do produto como um todo.

Projetos de software sem um conjunto bem definido de requisitos de qualidade tornam-se vulneráveis a falhas [3]. A quantificação dos níveis de requisitos de qualidade implica na escolha de métricas apropriadas e na definição de modelos de mensuração [4]. Os modelos devem mapear a realidade e/ou os requisitos pretendidos pelo usuário, enfocando as múltiplas questões referentes à construção do produto e monitorando possíveis desvios. Neste contexto, os modelos de avaliação da qualidade estão diretamente associados ao processo de medição, determinando como as medidas serão executadas e planejadas [5].

Vários modelos foram criados no sentido de propor um conjunto de critérios para avaliação de qualidade em software, por exemplo, os modelos de McCall [6], de Boehm [7], de Cavano e McCall [8]. Outros modelos podem ser citados, como os de Rocha [2] e o de Dromey [9].

A norma ISO/IEC 9126, conhecida no Brasil como NBR 13596 [10], surgiu como uma importante tentativa de consolidar as diferentes visões da qualidade em um modelo único, internacionalmente aceito.

Pensando nisso, buscamos com este trabalho pesquisar um modelo de qualidade voltado para o paradigma de orientação a objetos (OO). Nesse sentido, o Manual para Controle da Qualidade de Especificações Orientadas a Objetos [1] foi escolhido porque contém um rico conteúdo de características de qualidade aplicáveis ao contexto. Esse Manual considera para definição de características de qualidade e procedimentos para avaliação de software o modelo proposto por Rocha [2].

Apresentamos quatro estudos de casos em que esse modelo de avaliação de qualidade de sistemas de software orientados a objetos foi experimentado. Resultados preliminares sobre a aplicação desse modelo em um estudo de caso foram apresentados em Melo \& Rezende [11]. Este novo artigo, além de apresentar os resultados em estudos de casos complementares, apresenta também dados qualitativos e quantitativos sobre a aplicabilidade desse modelo. Este artigo também objetiva apresentar o ferramental construído para apoiar a aplicação desse modelo. Tal ferramental foi construído em função das necessidades identificadas na primeira aplicação desse modelo em [11].

Para atingir tal objetivo, nós organizamos este artigo da maneira descrita a seguir. $\mathrm{Na}$ seção 2, nós mostramos as principais características dos ambientes nos quais os estudos empíricos foram realizados. Então, na seção 3, o modelo de avaliação proposto por Beaufond [1] é analisado. Na seção 4, apresentamos a importância de se ter ferramentas de apoio durante o processo de avaliação para obter melhores resultados. Nós discutimos os principais resultados derivados desses estudos, tanto da avaliação dos projetos através do Manual, como também da aplicabilidade desse Manual durante o processo de avaliação, na seção 5. Finalmente, concluímos na seção 6 .

\section{$2 \quad$ Estudos de Casos}

Nesta seção, apresentaremos nas tabelas 1, 2, 3 e 4 algumas informações sucintas das características de cada estudo de caso, começando com as empresas e linguagem de desenvolvimento utilizada, as características das equipes e tempo de desenvolvimento dos projetos, fazendo, ainda, um resumo do número de critérios do Manual aplicados, quantos pacotes, classes, métodos e atributos tem cada projeto. Por fim, apresentaremos um resumo das documentações e acesso às informações disponíveis em cada estudo de caso avaliado. 
Os resultados dos primeiros estudos de casos poderiam ser melhores se a equipe fosse experiente no uso do manual, assim devem ser analisados com cuidado. Contudo, optamos por mostrar estes dados, visto que este é um problema que qualquer organização ira se confrontar caso deseje utilizar a abordagem citada. Ressalte-se também que o primeiro e o segundo estudo de caso foram avaliados sem nenhuma ferramenta de apoio. A partir das dificuldades percebidas, buscamos melhores recursos para o processo de avaliação. Para isso, realizamos treinamentos do Manual [1] com os participantes do processo de avaliação dos projetos e desenvolvemos uma ferramenta de apoio que será apresentada mais adiante, chamada SYSQUALITY. Desta forma, finalmente, armazenamos também nessa ferramenta os dois estudos de casos já avaliados antes de sua construção, para melhorar as informações finais desta pesquisa. Infelizmente, como acontece em qualquer projeto, à documentação de alguns dos projetos era muita restrita. Contudo, os avaliadores utilizaram os critérios que puderam ser aplicados a documentação existente.

Tabela 1 - Resumo dos Estudos de Casos por Sistemas e Linguagens Utilizadas

\begin{tabular}{|c|l|l|c|}
\hline Estudo & \multicolumn{1}{|c|}{ Empresa } & \multicolumn{1}{c|}{ Sistema } & Linguagem \\
\hline 1 & Assembléia Legislativa de Goiânia & Sistema de Gerenciamento de Consultas e Exames & Delphi 5 \\
\hline 2 & Trabalho de Pós-Graduação & Automação da Locadora de Vídeo VHS & Java \\
\hline 3 & Projeto da Empresa Oracle & $\begin{array}{l}\text { Sistema de Arrecadação Financeira da Vigilância } \\
\text { Sanitária }\end{array}$ & Java \\
\hline 4 & $\begin{array}{l}\text { Secretaria da Segurança Pública do } \\
\text { Estado de Goiás }\end{array}$ & $\begin{array}{l}\text { SAP - Sistema de Acompanhamento de Processos } \\
\text { do Procon }\end{array}$ & Java \\
\hline
\end{tabular}

Tabela 2 - Resumo dos Estudos de Casos por Equipe e Prazo

\begin{tabular}{|c|c|c|c|c|c|}
\hline Estudo & Analista Pleno & $\begin{array}{l}\text { Analista } \\
\text { Sênior }\end{array}$ & Programadores & Consultores 00 & $\begin{array}{l}\text { Tempo de } \\
\text { Desenvolvimento. }\end{array}$ \\
\hline 1 & 2 & 2 & 4 & 1 & 4 Meses \\
\hline 2 & 1 & 1 & 2 & 1 & 4 Meses \\
\hline 3 & indisponível & indisponível & indisponível & indisponível & indisponível \\
\hline 4 & 3 & 1 & 2 & 1 & 11 Meses \\
\hline
\end{tabular}

Tabela 2 - Resumo dos Estudos de Casos por Propriedades do Projeto Estudo Qtde_critérios_apl Qtde_Pacotes Qtde_Classe Qtde_Métodos Qtde_Atributos

\begin{tabular}{|r|c|c|c|c|c|}
\hline 1 & 136 & 0 & 9 & 51 & 69 \\
\hline 2 & 94 & 0 & 11 & 103 & 47 \\
\hline 3 & 104 & 15 & 112 & 290 & 289 \\
\hline 4 & 132 & 4 & 42 & 129 & 266 \\
\hline
\end{tabular}

Tabela 4 - Resumo dos Estudos de Casos por Documentação Disponível

\begin{tabular}{|c|c|c|c|c|c|}
\hline Estudos & $\begin{array}{c}\text { Documentação } \\
\text { Auxiliar }{ }^{1}\end{array}$ & $\begin{array}{c}\text { Caso de } \\
\text { Uso }\end{array}$ & $\begin{array}{c}\text { Diag. } \\
\text { Classe }\end{array}$ & $\begin{array}{c}\text { Diag. } \\
\text { Sequência }\end{array}$ & $\begin{array}{c}\text { Acesso a Equipe de } \\
\text { Desenvolvimento }\end{array}$ \\
\hline 1 & $\mathrm{X}$ & $\mathrm{X}$ & $\mathrm{X}$ & $\mathrm{X}$ & $\mathrm{X}$ \\
\hline 2 & $\mathrm{X}$ & $\mathrm{X}$ & $\mathrm{X}$ & $\mathrm{X}$ & \\
\hline 3 & & $\mathrm{X}$ & $\mathrm{X}$ & & \\
\hline
\end{tabular}

\footnotetext{
${ }^{1}$ Documentação Auxiliar: São documentações da análise de requisitos, contratos, textos explicativos, atas de reuniões, etc.
} 
O Manual identifica um conjunto de objetivos, fatores e subfatores da qualidade das especificações orientadas a objetos. Os objetivos apresentados no Manual foram definidos por Rocha [2]. São eles:

- Confiabilidade conceitual, dado que a especificação precisa satisfazer as necessidades e requisitos que motivaram sua construção.

- Confiabilidade da representação, que se refere às características de representação do produto que afetam sua compreensão e manipulação.

- Utilizabilidade, que determina a conveniência e a viabilidade de utilização da especificação ao longo do processo de desenvolvimento. Para que uma especificação seja utilizável são necessárias a confiabilidade conceitual e a confiabilidade da representação.

Segundo a autora, produtos de software são desenvolvidos para atenderem a determinadas necessidades de seus usuários. Após serem colocados em operação, espera-se que tenham uma vida útil, longa e produtiva. Para que isso se concretize, devem ser atingidos os objetivos de qualidade acima. Dessa forma, o Manual propõe vários atributos (critérios) de qualidade relacionados às especificações orientadas a objetos, considerando as características gerais de qualquer especificação que for gerada, independente do nível de abstração que a mesma represente. O modelo contém cento e quarenta e nove critérios de avaliação, divididos em quarenta e oito critérios do objetivo da confiabilidade da representação, setenta e sete critérios do objetivo da confiabilidade conceitual e vinte e quatros critérios do objetivo utilizabilidade.

O método para avaliação da qualidade utilizado nesse manual foi proposto por Rocha [2], e baseia-se nos seguintes conceitos:

- Objetivos de Qualidade: são propriedades gerais que o produto deve possuir.

- Fatores de Qualidade do Produto: determinam a qualidade do produto sob o ponto de vista dos diferentes usuários do produto (usuário final, mantenedores, etc.).

- Critérios: são atributos primitivos possíveis de serem avaliados;

- Processo de Avaliação: determina o processo e os instrumento a serem utilizados para se medir o grau de presença, no produto, de um determinado critério.

- Medidas: indica o grau de presença, no produto, de um determinado critério.

- Medidas Agregadas: são o resultado da agregação das medidas obtidas na avaliação segundo os critérios que quantificam os fatores.

\subsection{Estrutura do Manual}

No Manual, são descritos os procedimentos necessários para realizar as avaliações de especificações segundo o método, sendo que para cada objetivo de qualidade, são definidos os fatores e subfatores de qualidade a eles relacionados. Portanto, para cada critério foram descritos:

- Definição: é apresentada uma definição precisa e explícita do critério.

- Técnica de Avaliação: método de controle da qualidade sugerido para realizar a avaliação. 
- Processo de Avaliação: o processo de avaliação adotado mede o grau de presença de cada critério em um intervalo de 0 a 1 , utilizando uma escala ordinal como indicado na Tabela 5. A variável $\mathrm{V}_{\mathbf{x}}$, utilizada em alguns processos de avaliação representa valores de aceitação estabelecidos ao longo do desenvolvimento do projeto e/ou considerando a experiência da organização no desenvolvimento de sistemas orientados a objetos.

- Sugestões para Correção: são indicadas medidas corretivas quando pertinentes.

- Atributos de Qualidade Relacionados: são identificados os atributos de qualidade relacionados ao critério que está sendo avaliado, explicando brevemente o motivo desse relacionamento.

\section{Tabela 5 - Graus de Presença do Critério - Escala Ordinal}

\begin{tabular}{|c|c|l|}
\hline ESCALA & EQUIVALÊNCIA & INTERPRETAÇÃO \\
\hline 1 & Total presença & Indica que não há dúvida que o critério está totalmente presente. \\
\hline 0,75 & Alta Presença & Indica um alto grau de presença do critério, mas não total. \\
\hline 0,50 & Moderada Presença & Indica um grau de presença aceitável do critério. \\
\hline 0,25 & Baixa Presença & $\begin{array}{l}\text { Indica um baixo grau de presença do critério, sendo necessário o uso } \\
\text { de medidas corretivas. }\end{array}$ \\
\hline 0 & Total Ausência & Indica de maneira absoluta que o critério está ausente. \\
\hline
\end{tabular}

\section{Considerações ao utilizar o Manual:}

- Ao avaliar cada critério, é importante observar qual a técnica que está sendo utilizada na avaliação, ou seja, se é a técnica de inspeção individual ou a de reunião de inspeção.

- Observar qual o subproduto utilizado na avaliação do critério.

- Dependendo do projeto avaliado, algumas características sugeridas pelo Manual podem ser modificadas ou complementadas.

- A característica que demonstra os atributos de qualidades relacionados ao critério de avaliação que está sendo avaliado é bastante importante para a compreensão do projeto como um todo, ou seja, ela ajuda a equipe de desenvolvimento a entender como um determinado processo implicará na importância da qualidade de outros processos relacionados.

\section{$4 \quad$ Ferramentas de Apoio à Avaliação}

Durante a avaliação do primeiro projeto, houve uma grande dificuldade para utilizar alguns critérios propostos no Manual, devido à falta de ferramentas que nos auxiliassem na manipulação dos critérios e seus resultados. Outro problema detectado foi a dificuldade de visualizar os resultados e seus relacionamentos com os atributos de qualidade, os produtos ou artefatos envolvidos e as técnicas de inspeções utilizadas. Vimos, também, a necessidade de uma análise geral do Manual, juntamente com todas as suas características. Dessa forma, tornou-se necessário encontrar ou criar uma ferramenta de apoio para a realização das avaliações durante a aplicação dos estudos de casos. Criamos, então, a ferramenta SYSQUALITY.

Com ela, pudemos cadastrar os objetivos de qualidade, juntamente com seus fatores e subfatores e configurar todo o Manual para ser aplicado em diversos projetos. Uma das 
grandes vantagens dessa ferramenta é a de possibilitar que o responsável pela avaliação possa escolher somente os critérios necessários, montando um modelo específico aos seus objetivos.

Após montar seu próprio modelo, este também pode ser utilizado por diversos projetos semelhantes. Dessa forma, o avaliador ganha agilidade no processo de avaliação.

Segue uma breve descrição da ferramenta SYSQUALITY com seus módulos principais:

1 - Módulo de Tabelas: Este módulo tem como objetivo o cadastramento de vários itens que envolvem o processo de avaliação da qualidade de software. Sendo eles os seguintes:

- Técnicas de Avaliação - Cadastra-se as técnicas de avaliação que podem ser utilizadas para cada critério. Assim, ao cadastrar o modelo de qualidade selecionado, informa-se qual a técnica de avaliação mais adequada para cada critério desse modelo.

- Atributos de Qualidade - Cadastra-se os atributos de qualidades que estão envolvidos em um determinado critério. Ex. No Objetivo Confiabilidade da Representação, Fator Manipulabilidade e Sub_fator Disponibilidade, tem-se o critério da Acessabilidade, que possui características que avaliam se qualquer usuário autorizado pode facilmente consultar a especificação e/ou obter uma cópia da mesma. Este critério envolve segundo Beaufond [1], os atributos de qualidade Avaliailidade, Manutenibilidade e Reutilizabilidade.

- Produtos ou Artefatos - São cadastrados quais os produtos que são necessários para a realização da avaliação de cada critério. Ex. No Objetivo Confiabilidade da Representação, Fator Comunicabilidade e Sub-fator Correção no Uso do Método, temse o critério Correção Semântica (CSM), que avalia se o conjunto de regras semânticas, pré-definidas no método de especificação, foi usado corretamente. Este critério segundo Beaufond [1], necessita do Sub-produto Modelo de Classe-objeto.

- $\mathbf{G Q M}^{2}$ - São cadastrados os GQM's que serão aplicados para a avaliação da aplicabilidade do modelo utilizado. Definindo o objetivo do GQM, as questões que envolvem cada objetivo e a forma de obter os resultados através de uma escala de interpretação das questões levantadas.

- Escala do GQM - São cadastrados vários tipos de escalas de interpretação de determinadas questões. Ex. Uma questão que deseja saber se um determinado critério é difícil de ser aplicado foi utilizada uma escala de: Muito Difícil, Difícil, Médio, Fácil e Muito Fácil.

2 - Módulo Caracterize: Este módulo tem como objetivo cadastrar todos os itens que envolvem o modelo de qualidade a ser montado, bem como caracterizar os critério que são mais adequados a um determinado projeto que será avaliado, isto é, neste módulo podem ser selecionados somente os critérios necessários a um determinado projeto, levando em consideração o ambiente de desenvolvimento, ferramentas utilizadas e o perfil dos desenvolvedores.

- Objetivo - São cadastrados os objetivos, fatores e sub-fatores de qualidade do modelo selecionado.

- Critérios - São cadastrados os critérios e todos os itens que envolvem o mesmo, tais como: produto necessário para avaliação, técnica de inspeção necessária, os atributos de qualidades que estão envolvidos, bem como, as questões que serão analisadas para este critério e a forma de obter essa medida, através de uma métrica ou um valor que represente o resultado obtido.

\footnotetext{
${ }^{2}$ Goal/Question/Metric [14]
} 
- Montar o Modelo - Neste item é que o avaliador pode montar um modelo específico à sua avaliação, selecionando somente os critérios mais importantes do modelo já cadastrado.

3 - Módulo Execute: Neste módulo são realizadas as avaliações dos projetos.

- Cadastrar Projeto - Cadastra-se o projeto que será avaliado, informando o nome da empresa, o método utilizado, os tipos de documentações disponíveis (artefatos), a linguagem de programação utilizada, a quantidade de pacotes, classes, métodos e atributos desse projeto.

- Aplicar Questionário - Aqui será selecionado o projeto a ser avaliado, o modelo específico a avaliação criado no módulo anterio que será aplicado e qual GQM será utilizado para avaliação da aplicabilidade do modelo. E, finalmente, será realizada a avaliação do modelo em si.

4 - Módulo Análise: Neste módulo são montados os resultados das análises, tanto do projeto avaliado quanto da aplicação do GQM sobre o modelo utilizado.

Outro problema encontrado foi a dificuldade para obter certas medidas de qualidade, principalmente as voltadas à complexidade da estrutura de projetos OO. Devido ao pouco tempo disponível, buscamos uma outra ferramenta que pudesse ser utilizada como apoio para obter tais medidas. A ferramenta escolhida foi a JMETRIC, um analisador de métricas para programas em JAVA, desenvolvido por Cain [12]. Essa ferramenta auxiliou no processo de avaliação de algumas métricas do projeto.

\section{$5 \quad$ Resultados}

Nesta seção, descreveremos os principais resultados das avaliações dos critérios do modelo proposto sobre os projetos avaliados, bem como os resultados das análises levantadas sobre a aplicabilidade do Manual durante o processo de avaliação, juntamente com algumas explicações dos valores encontrados. Em Rezende [13], pode ser encontrada uma descrição detalhada desses resultados. Aqui, devido a problemas de espaço, somente serão discutidos os resultados de forma agregada. Em Rezende [13], é mostrado o resultado da avaliação de todos os critérios pospostos no manual de avaliação.

\subsection{Análise dos Resultados dos Objetivos e Fatores de Qualidade Por Estudo de Caso}

Nesta seção, serão mostrados os resultados das avaliações dos estudos de casos realizados, apresentando os aspectos importantes que foram detectados nos projetos, através da aplicação do Manual. Com a identificação dos itens problemáticos nas especificações do projeto, o gerente do projeto poderá (se ainda for possível) conduzir atividades de testes e corrigir esses problemas que poderão prejudicar o sistema, ou, se não houver mais tempo, evitar tais problemas em projetos posteriores.

Os objetivos de qualidade são as propriedades gerais que o produto de software deve possuir. Dessa forma, podem ser observados os resultados das avaliações nos estudos de casos apresentados de um modo mais sintético.

Através da Figura 1, observou-se que no objetivo da Confiabilidade Conceitual, os projetos que estão com a melhor especificação dos requisitos dos clientes foram os estudos de caso 01 e 03 , que alcançaram $60 \%$ e $63 \%$ dos valores ideais para esse objetivo de qualidade. Já os estudos de casos 02 e 04 tiveram $47 \%$ e $32 \%$ dos valores ideais. 
No objetivo da Confiabilidade da Representação, que são as características de representação do produto e que afetam a sua compreensão e manipulação, os resultados foram mais preocupantes em todos os projetos, principalmente nos estudos de casos 02,03 e 04 , que alcançaram somente $37 \%, 43 \%$ e $38 \%$, respectivamente, dos valores ideais.

Já no objetivo da Utilizabilidade, que determina a viabilidade de utilização da especificação ao longo do processo de desenvolvimento, nos estudos de casos 02 e 03 não foi possível avaliar os resultados, devido à falta de documentação e acesso às pessoas responsáveis pelos projetos. Assim, para o gerente de projeto ter uma visão melhor desses resultados, ele precisa analisar cada um desses objetivos mais detalhadamente, a fim de identificar melhor os problemas encontrados.

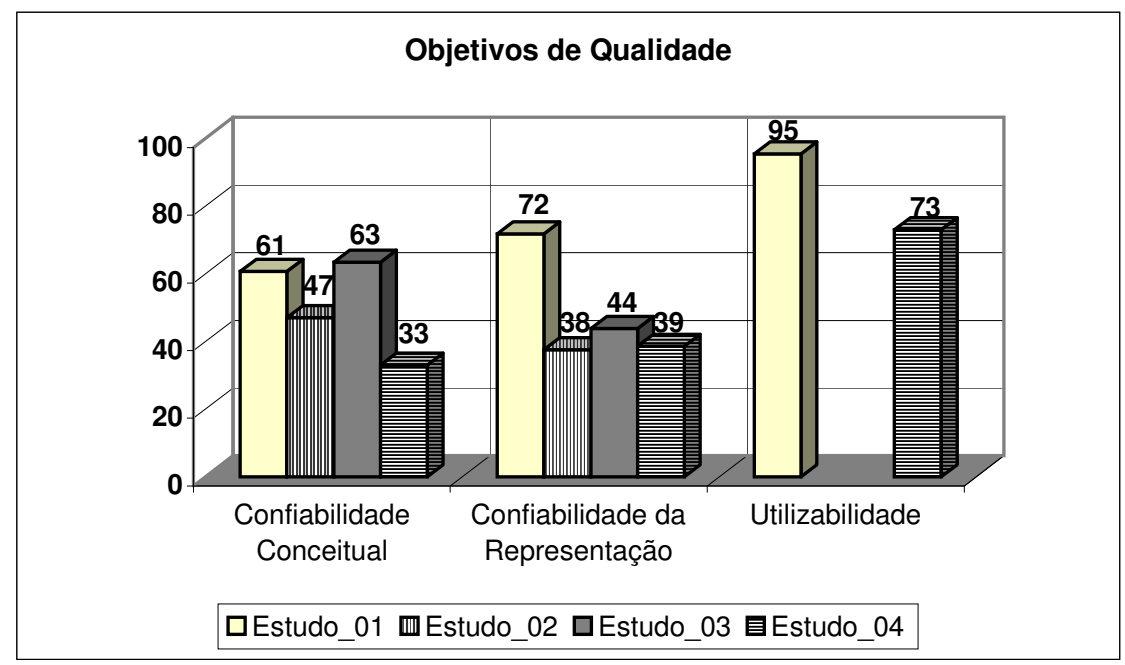

Figura 1- Resultados dos Objetivos de Qualidade por Estudo de Caso

Os fatores de qualidade determinam a qualidade do produto e os instrumentos a serem utilizados para se medir o grau de presença, no produto, de um determinado critério.

Nos estudos de casos apresentados na Figura 2, o primeiro objetivo é o da Confiabilidade Conceitual, que abrange dois fatores de qualidade: Fidedignidade e Suficiência. Na Fidedignidade, os estudos de casos 01, 02 e 03 apresentaram um resultado maior que $50 \%$ e menor que $60 \%$ dos valores ideais. Já no estudo 04 , apenas $26 \%$ dos resultados obtiveram o valor desejado, deixando claro que as necessidades e expectativas dos usuários do produto avaliado provavelmente não estão sendo atendidas.

Já para o fator Suficiência, o estudo de caso que mais chamou a atenção foi o 02 , por alcançar somente $27 \%$ do valor desejado. Nesse caso, provavelmente não estão presentes nas especificações somente os aspectos necessários ao projeto.

Os fatores de qualidade do objetivo da Confiabilidade da Representação são: Comunicabilidade e Manipulabilidade.

No fator Comunicabilidade, foram alcançados mais de $50 \%$ dos valores ideais, em todos os projetos. Quanto ao fator Manipulabilidade, que avalia a facilidade de manipulação da especificação para diversas formas de uso, os valores foram muito preocupantes, em todos os projetos, principalmente nos estudos 02 e 03.

Por último, o objetivo da Utilizabilidade tem o fator Implementabilidade, que avalia se uma especificação de requisitos pode ser implementada. Nos projetos 02 e 03 não foi possível avaliar esse fator. 


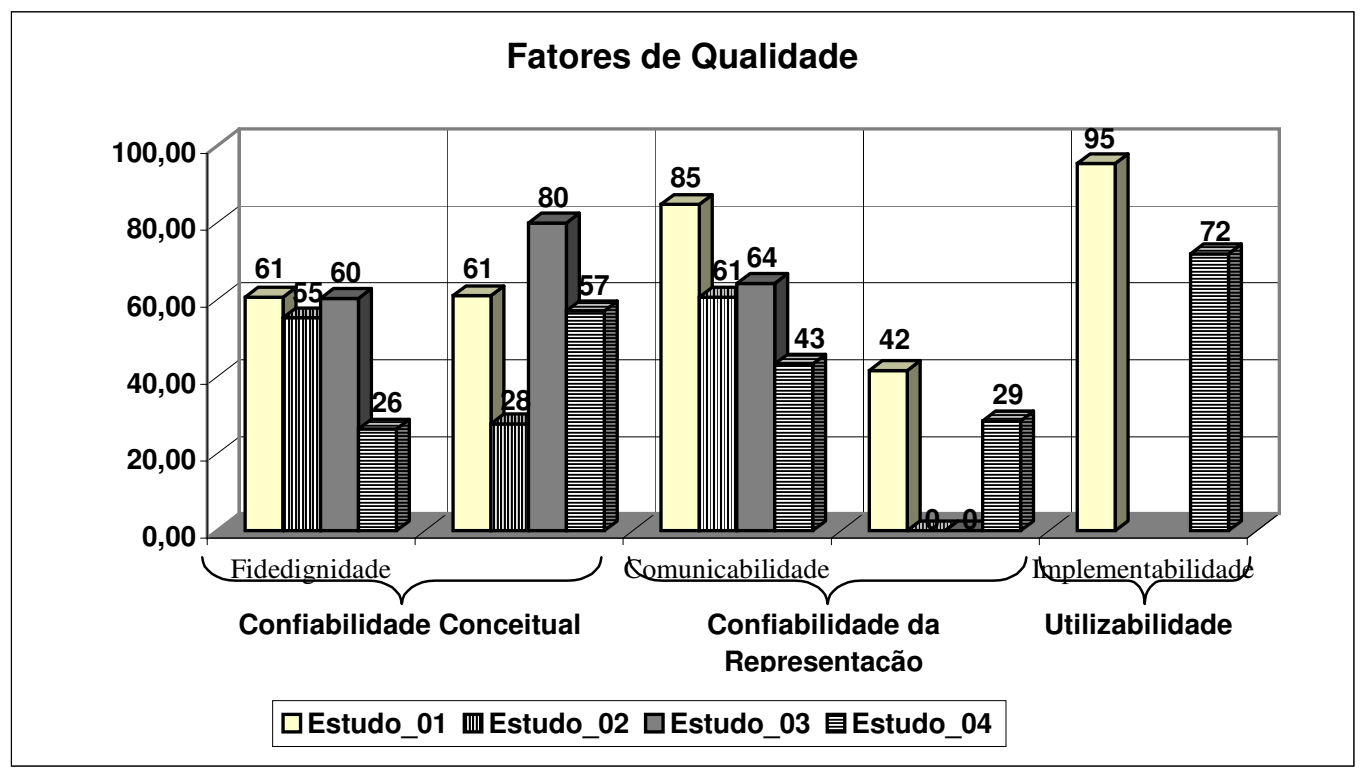

Figura 2 - Resultados dos Fatores de Qualidade por Estudo de Caso

Os resultados apresentados nos fatores de qualidade acima ainda não são suficientes para que o gerente de projeto tome as decisões necessárias a fim de aprimorar o projeto avaliado. As análises podem ser realizadas de uma forma mais detalhada, através dos resultados das avaliações dos subfatores de qualidade definido no Manual. Devido aos problemas de espaço, somente serão discutidos neste documento os resultados dos objetivos e dos fatores de qualidades.

\subsection{Análise das Métricas da Complexidade Estrutural do Projeto OO}

Durante o estudo do Manual, observamos que vários critérios necessitavam da definiçãao de uma variável Vx. Ela representa os valores de aceitação estabelecidos ao longo do desenvolvimento do projeto, considerando a experiência da organização no desenvolvimento de sistema orientado a objeto. Através da variável Vx, o avaliador conduz o resultado da avaliação, identificando o resultado aceitável, ou não, do critério. Essa é a principal diretriz a ser providenciada quando utilizamos métricas para avaliar o produto de software. Devemos identificar os valores que são aceitáveis e não aceitáveis, para efetuar ações corretivas com os valores inaceitáveis. Vimos, então, a necessidade de estudar os efeitos dessa variável Vx, mais conhecida como indicadores de limites (Thresholds).

Nos estudos de casos aplicados neste trabalho foram definidos os valores da variável Vx, proposta no Manual, através de algumas análises estatísticas (média, moda, mediana, variância e desvio padrão). Isso porque em nenhum dos estudos de casos aplicados havia uma equipe com bastante experiência para definir esses valores. Os valores de percentuais sugeridos por alguns pesquisadores não foram utilizados, por não se saber, ao certo, quão dispersos estavam os nossos dados de cada medida. Dessa forma, ao serem calculados a média, mediana, moda, variância e o desvio padrão de cada métrica, eram definidos os valores das variáveis Vx (thresholds) de acordo com os resultados dessas análises.

Os critérios mais difíceis de serem avaliados durante os estudos de casos foram os relacionados à complexidade estrutural do projeto orientado a objeto. Esses critérios necessitam dessa variável $\mathrm{Vx}$. 
As métricas da complexidade estrutural, dependendo do tamanho do projeto, e se forem obtidas sem nenhuma ferramenta de apoio, podem tomar bastante tempo dos avaliadores e, conseqüentemente, inviabilizar a avaliação. $\mathrm{O}$ esforço de inspeção e controle do projeto não pode ultrapassar o esforço de desenvolvimento do mesmo. Por esse motivo, sugerimos que, ao escolher um modelo que utiliza métricas em seus critérios de avaliação, é importante procurar uma ferramenta que possa auxiliar essa tarefa, ou mesmo desenvolver uma que atenda a essas necessidades. A compreensão da complexidade do projeto é fundamental para avaliar se ele foi bem definido, e o quanto de esforço provavelmente será necessário para manutenção e reutilização dos componentes definidos nesse projeto.

Outro ponto importante que deve ser analisado é a maneira como o projeto foi estruturado. Ao avaliar a variável $\mathrm{Vx}$, talvez seja interessante separar as métricas por camadas, uma vez que a complexidade da camada de interface é totalmente diferente da camada de negócio. Assim, ao se calcularem as estatísticas sugeridas neste trabalho, aconselhamos que sejam também realizados os cálculos dessas variáveis $\mathrm{Vx}$, separando-as em suas camadas, para definir assim uma variável Vx (threshold) mais próxima à realidade do projeto.

Através dos resultados dessas métricas, pode ser identificada a utilização incorreta do paradigma de orientação a objetos. Como exemplo, podemos destacar o estudo de caso 04, no qual foi constatada a falta de experiência dos desenvolvedores com a metodologia adotada. A forma representacional de muitas classes definidas nesse projeto estava errada, utilizando mais uma forma funcional adotada na abordagem estruturada, em vez da forma correta da orientação objeto.

Nos estudos de casos, detectou-se a grande dificuldade da utilização correta da abordagem orientada a objeto, devido à falta de experiência com a mesma em projetos anteriores.

O Manual é de grande utilidade para ajudar a assimilar melhor essas novas características. Em cada critério avaliado, são levantadas discussões sobre o assunto analisado, bem como sobre os atributos de qualidades que são afetados por esse critério. E, em alguns casos, são apresentadas sugestões de correções para problemas encontrados.

As pessoas que estiverem envolvidas no processo de avaliação da qualidade das especificações OO estão, com certeza, mais preparadas para definir novos projetos. Elas adquirem o conhecimento da importância da definição correta das notações propostas na metodologia e, principalmente, da organização e padronização da documentação gerada.

\subsection{Análise dos Resultados da Aplicabilidade do Manual}

Para auxiliar na avaliação do Manual, recorremos à abordagem GQM para definir objetivos e questões que respondessem as nossas necessidades. Através dessas questões, podemos avaliar os resultados das opiniões sobre a aplicabilidade do Manual e guardar essas informações para serem aproveitadas em projetos futuros.

Outro aspecto importante foi adequar as questões definidas no GQM para serem aplicadas a cada critério do Manual. Assim, conseguimos levantar informações tanto na estrutura do GQM definido quanto na estrutura proposta no Manual. Além disso, conseguimos comparar as evoluções e mudanças relevantes ocorridas entre as avaliações de cada estudo de caso.

Após definirmos o GQM, adaptamos tal recurso à ferramenta SYSQUALITY. Apesar de serem avaliações empíricas, conseguimos definir um segmento melhor para nossas avaliações da aplicabilidade do Manual, durante o processo de avaliação. 
Assim, definimos em nosso GQM três objetivos específicos para avaliar a aplicabilidade do Manual:

- Esforço para aplicar o modelo.

- Identificação de problemas e prevenção.

- Esforço para entender o modelo.

Através desses objetivos, definimos as questões levantadas para cada critério que estava sendo avaliado. As métricas disponíveis para as respostas dessas questões foram definidas, não de forma quantitativa, devido à subjetividade dessas respostas, dependendo muito do ponto de vista e experiência do avaliador. Com isso, podemos dizer que nos resultados da aplicabilidade do GQM podem ocorrer variações, de acordo com as pessoas responsáveis pela avaliação.

Segue abaixo as questões e as respostas disponíveis no GQM definido:

- Esforço para Aplicar o Modelo

- Qual o grau de dificuldade para obter a medida do critério analisado?

- Muito Difícil.

- Difícil.

- Médio.

- Fácil.

- Muito Fácil.

- Qual o tempo gasto para analisar cada critério?

- Mais de 3 horas.

- Entre 01h e $31 \mathrm{~m}$ a 3 horas.

- Entre $31 \mathrm{~m}$ e $1 \mathrm{~h}$ e 30 minutos.

- Entre $11 \mathrm{~m}$ e 30 minutos.

- Até 10 minutos.

- Identificação de problemas e Prevenção

○ Qual a taxa de defeitos em média descoberta no projeto?

- Nenhuma.

- Poucas.

- Regular.

- Algumas.

- Muitas.

- Qual o grau de necessidade do critério para o projeto?

- Nenhuma.

- Pouca.

- Média.

- Importante.

- Indispensável.

- Esforço para entender o Modelo

○ Qual o esforço para entender o critério avaliado?

- Muito Difícil.

- Difícil.

- Médio.

- Fácil.

- Muito Fácil.

Os resultados dessas análises estão representados através da Figura 3, Figura 4 e Figura 5, juntamente com os comentários sobre todo o processo envolvido. Como as informações obtidas do GQM foram armazenadas em uma base de dados, podem ser 
visualizadas as correlações desses resultados de diversas formas, dependendo da criatividade do avaliador em desenvolvê-las.

Esses resultados foram avaliados para cada estudo de caso e pelos objetivos de qualidade definidos no Manual. Também podem ser identificados vários tipos de informações que auxiliam ao gerente do projeto a definir quais os critérios mais adequados para determinados projetos, dependendo do objetivo da avaliação.

Seguem alguns exemplos de informações que podem ser obtidas através da base de dados gerada pelo sistema:

- Quais foram os critérios que identificaram mais problemas de um determinado projeto? Ou de todos os projetos?

- Quais são os critérios mais demorados para serem avaliados?

- Quais são os critérios mais difíceis de serem avaliados?

- Quais os critérios que não são necessários para o projeto alvo? Em qual fator de qualidade eles estão mais acentuados?

- Entre outras.

\subsection{Análise Geral da Aplicabilidade do Manual}

Na Figura 3, Figura 4 e Figura 5, temos os resultados gerais dos seguintes objetivos:

- Esforço para Aplicar o Modelo

- Qual o grau de dificuldade para obter a medida do critério analisado?

- Qual o tempo gasto para analisar o critério?

- Identificação de Problemas e Prevenção

- Qual a taxa de defeitos, em média, descoberta no projeto?

- Qual o grau de necessidade do critério para o projeto?

- Esforço para Entender o Modelo

- Qual o esforço para entender o critério avaliado?

Qual o grau de dificuldade para obter a medida do critério analisado?

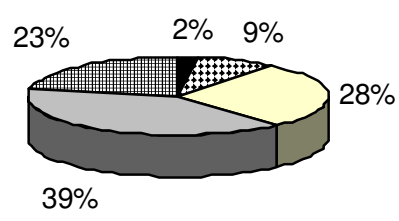

Muito Difícil 国 Difícil $\square$ Médio $\square$ Fácil 团 Muito Fácil
Qual o tem po gas to para analisar o critério?

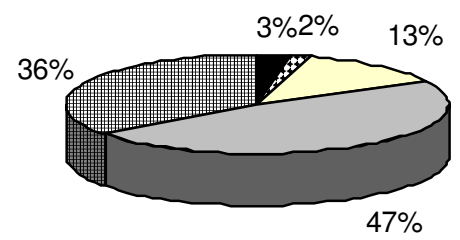

Mais de 3 horas

Entre 00:31 a 01:30 h

Até 00:10 Minutos 
Qual a taxa de defeitos em média descoberta no

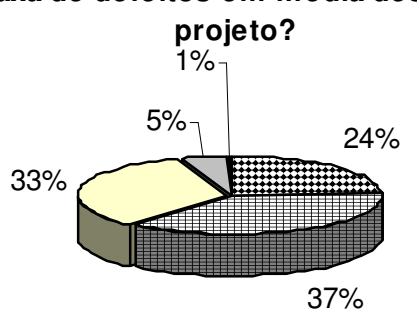

国Nenhuma 国Poucas $\square$ Regular $\square$ Algumas $\square$ Muitas
Qual o grau de necessidade do critério para o

projeto?

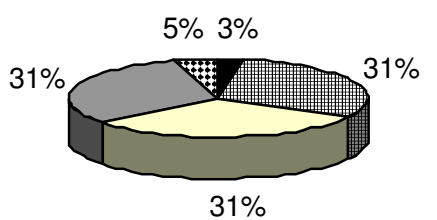

Nenhuma 毘Pouca $\square$ Média $\square$ Importante Indispensável

Figura 4 - Resultado Geral da Identificação de Problemas e Prevenção

Qual o esfoço para entender o critério avaliado?

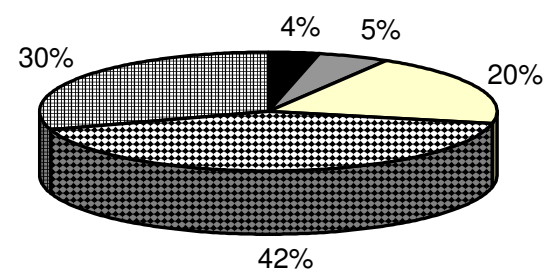

Muito Difícil $\square$ Difícil $\square$ Médio 国Fácil 田Muito Fácil

Figura 5 - Resultado Geral do Esforço para entender o Modelo

\section{Vantagens:}

- Mais de 50\% dos critérios definidos no Manual são fáceis ou muito fáceis de serem avaliados;

- $80 \%$ dos critérios necessitaram, em média, menos de 30 minutos para serem aplicados, dependendo diretamente do tamanho do projeto;

- Mais de $70 \%$ dos critérios são fáceis ou muito fáceis de serem entendidos pelo avaliador, lembrando que, a cada novo estudo de caso avaliado pela mesma equipe, esse índice tende a aumentar.

\section{Dificuldades:}

- $\quad 20 \%$ em média dos critérios não puderam ser avaliados. Isso ocorreu devido a algumas características dos projetos. Por exemplo: se o projeto não possuía determinados diagramas em sua documentação pela não-necessidade dos mesmos, os critérios que avaliavam esses diagramas não puderam ser avaliados.

- Apenas $31 \%$ dos critérios foram considerados importantes para os avaliadores, e 5\% indispensáveis de serem avaliados.

- A questão sobre taxa de defeitos obteve mais de $70 \%$ dos resultados, com poucos ou nenhuns erros encontrados. Esse resultado ocorreu porque várias questões levantavam a presença de defeitos no projeto. Porém, a ausência do mesmo não implicava em um erro, e sim em possíveis problemas futuros. Dessa forma, para essa questão, consideramos apenas os erros realmente identificados.

Esses valores tendem a mudar quando analisados por estudo de caso, em que a documentação disponível, o tamanho do projeto, as experiências com o Manual e a equipe de desenvolvimento do projeto se refletem diretamente nos resultados obtidos no GQM definido. 
Numa visão geral, com nossa experiência das avaliações realizadas, vimos que o Manual tem grande utilidade, principalmente para os gerentes de projetos que desejam obter o melhor aproveitamento da abordagem, melhorando a produtividade no desenvolvimento de projetos de software.

A forma como as questões estão apresentadas também pode refletir na agilidade do processo de avaliação, pois vários critérios poderiam ser avaliados por equipes diferentes, principalmente nos objetivos da confiabilidade conceitual e da representação, podendo, nesse caso, talvez até ajudar nos resultados finais. Um projeto pode ter suas especificações corretas de acordo com o método de desenvolvimento adotado, mesmo não atendendo aos requisitos desejados pelo cliente. Dessa forma, o avaliador não seria influenciado por um resultado excelente ou ruim em um determinado objetivo de qualidade para outro objetivo.

A necessidade dos critérios está diretamente relacionada ao porte do projeto e à experiência da equipe de desenvolvimento. Assim, ficou bem claro que qualquer modelo de qualidade, antes de ser aplicado, necessita de um estudo inicial para que o moderador ou responsável pela avaliação possa selecionar somente os critérios de qualidades que serão importantes para o projeto, agilizando e melhorando o objetivo final da avaliação.

\section{6}

Conclusões

Nós apresentamos, neste artigo, quatro estudos de casos nos quais utilizamos um modelo de referência para avaliação da qualidade de sistemas de software orientados a objetos, com objetivo de verificar sua aplicabilidade. Tal modelo é denominado Manual para Controle da Qualidade de Especificações Orientadas a Objetos, proposto em Beaufond [1]. Ele adere ao método de avaliação proposta por Rocha [2]. Definimos um GQM, para obter informações relevantes a respeito dos benefícios e dificuldades encontrados durante o processo de avaliação dos projetos.

Este Manual é fortemente baseado em medidas de software orientadas a objetos. Ele também incorpora sugestões técnicas em relação ao uso dos conceitos fornecidos por metodologias de desenvolvimento de sistemas orientadas a objetos (OO). Ou seja, uma vez que o sistema é mensurado, diretrizes são fornecidas de como interpretar as medidas, permitindo aos desenvolvedores e gerentes tomarem ações corretivas, quando necessário. As métricas avaliam desde o fato de a empresa contratante ser capaz de assumir as despesas do projeto e de a contratada contar com profissionais qualificados para o desenvolvimento, até o nível de abstração de cada classe definida no projeto avaliado.

A utilização do Manual, em nossos estudos de casos, comprovou a quantidade de informações para a tomada de decisões que podem ser geradas com sua utilização, justificando sua importância, principalmente nos projetos que foram avaliados durante seu processo de desenvolvimento. Porém, o ganho não fica somente no projeto em si, mas também no enriquecimento do conhecimento técnico que a equipe responsável pela avaliação obtém a cada projeto avaliado.

Entretanto, alguns cuidados precisam ser tomados antes da utilização do Manual:

- Selecionar uma equipe com bastante experiência no método de desenvolvimento orientado a objetos, para conduzir as avaliações.

- Fornecer treinamento a respeito de todos os critérios do Manual antes da avaliação.

- Procurar ferramentas de apoio ao processo de avaliação;

- Selecionar somente os critérios necessários na avaliação de um determinado projeto. Isso pode ser feito através de algumas características do projeto, tais como: técnicas de desenvolvimento do software, domínio da aplicação, 
características da equipe de desenvolvimento, tamanho e complexidade do projeto, ou até mesmo documentação disponível.

Esta foi a nossa experiência. A participação em quatro projetos reais, utilizando o Manual para Controle da Qualidade de Especificações Orientadas a Objetos [1], podendo vivenciar e refletir sobre os problemas que ocorreram, tanto no desenvolvimento de software orientado a objeto quanto durante o processo de avaliação da qualidade dos mesmos.

\section{Referências}

[1] Clifton E. Beaufond Clunie. Avaliação da Qualidade de Especificações Orientadas a Objetos, Rio de Janeiro, RJ, Tese de Doutorado, COPPER/UFRJ, Março, 1997.

[2] Ana Regina C. da Rocha, Um Modelo para Avaliação da Qualidade de

[3] Boehm, B., HOH, I., Identifying Quality-Requirement Conflits, IEEE Software, 13(2): 25, March, 1996.

[4] Mainini T. Billot L., PERFIDE: an environment for evaluation and monitoring of software reliability metrics during the test phase, This paper appears in: Software Engineering Journal, CODEN: SEJOED, 5(1), 27-32, Jan, 1990.

[5] Olivé A., Sancho, M. R., Validating Conceptual Specifications through Model Ececution, Information Systems, 21(2):167-186, 1996.

[6] MCCALL, J. A., Richards, P. g., and Walters, G. F., 1977, Factors in Software Quality, Vols. I, II, and III (NTIS AD/A-049/015/055), NTIS, Springfield, VA, 1977.

[7] Boehm, et al. B. W., J. R. Brown H Kaspar, M. Lipow, Gl J. Machleod, M.J.Merrritt, Characteristics of Software Quality. 1978.

[8] J. ${ }^{\text {a }}$ Mccall, J. P. Cavano,A Framework for the Measurement to Software Quality. Em Proc. of the ACM Software Quality Assurance Workshop, Nov., 1978.

[9] G. Dromey, Cornering the Chimera, Em IEEE Software, janeiro, 13(1): 33-43, 1996. Especificações, Tese de D. Sc, PUC-RJ, Rio de Janeiro, RJ, Brasil, 1983.

[10] NBR 13596. Tecnologia de produto de software. Características de qualidade e diretrizes para o seu uso. ABNT, 1996.

[11] Walcélio L. Melo e Denia Kuhn Rezende, Avaliação de Sistemas Software Orientados a Objetos: Um Estudo de Caso, CITIS 2002, Curitiba, PR, Brasil, 2002.

[12] Andrew Cain, JMETRIC, School of Information Technology at Swinburne University of Technology, http://www.it.swin.edu.au/projects/jmetric/default.htm, April, 1998.

[13] Denia Kuhn Rezende, Avaliação da aplicabilidade de um modelo de qualidade para especificações de projetos orientados a objetos, Dissertação, UCB-BR, Brasília, DF, Brasil, Março, 2003.

[14] Egon Berghout \& Rini Van Solingen, Goal/Question/Metric Method, A Practical Guide for Quality Improvement of Software Development, , McGraw-Hill, 1999. 